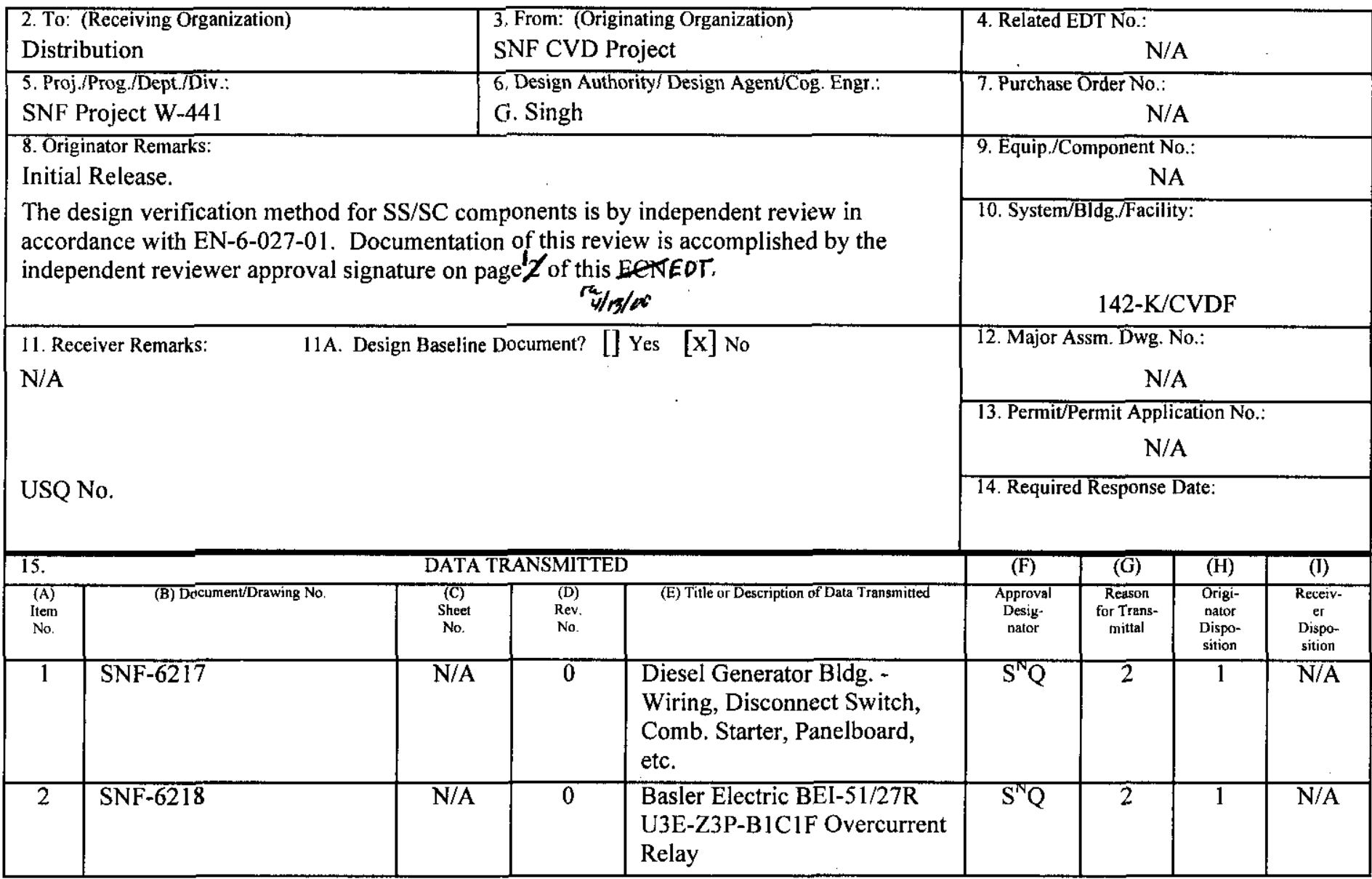

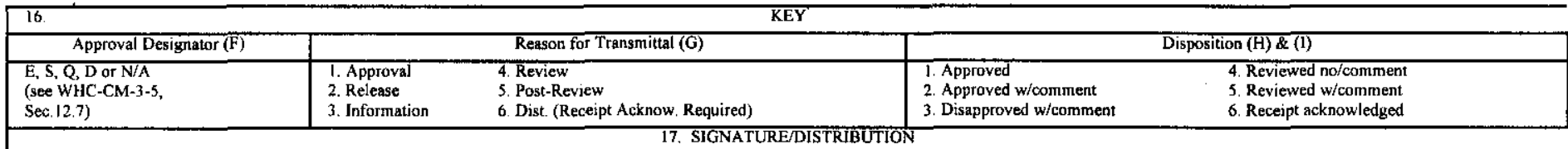

(See Approval Designator for required signatures)

\begin{tabular}{|c|c|c|c|c|c|}
\hline $\begin{array}{l}\text { (G) } \\
\text { Rea- } \\
\text { son }\end{array}$ & $\begin{array}{l}\text { (H) } \\
\text { Disp. }\end{array}$ & $\begin{array}{llll}\text { (J) Name } & \text { (K) Signature } & \text { (L) Date } & \text { (M) MSIN } \\
& \end{array}$ & $\begin{array}{l}(\mathrm{G}) \\
\text { Rea- } \\
\text { son }\end{array}$ & $\begin{array}{c}(\mathrm{H}) \\
\text { Disp. }\end{array}$ & $\begin{array}{llll}\text { (J) Name } & \text { (K) Signature } & \text { (L) Date } & \text { (M) MSIN }\end{array}$ \\
\hline 2 & 1 & Design Authority & & & See Distribution \\
\hline 2 & 1 & Engineer C. Van & & & \\
\hline 2 & 1 & Rep Mgr. & & & \\
\hline 2 & 1 & QA H. Chaffin & & & \\
\hline 2 & 1 & 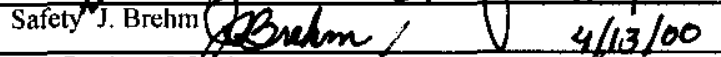 & & & usQ-like:Cvo-00-0/82823 4/13/00 \\
\hline 2 & 1 & Ind. Review J. Itwin & & & * Approval authorized, parallel preparation of USQ \\
\hline & & ${ }^{*}$ C. Haller & & & Screening with implementation of ECN per NSDI-02. \\
\hline
\end{tabular}
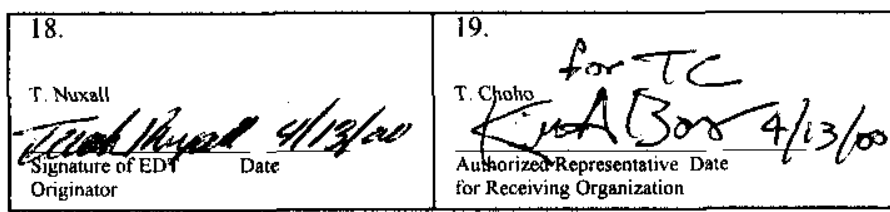

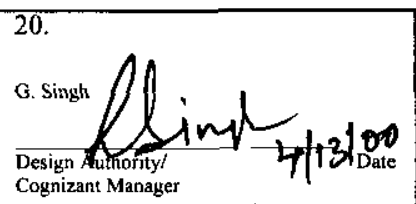

21. DOE APPROVAL (if required)

Ctrl. No.

[] Approved

[] Approved w/comments

[] Disapproved w/comments

BD-7400-172-2 (05/96) GEF097 
SNF-6218

Revision 0

\section{Basler Electric BEI-51/27R \\ U3E-Z3P-B1C1F \\ Overcurrent Relay}

Prepared for the U.S. Department of Energy

Assistant Secretary for Environmental Management

Project Hanford Management Contractor for the

U.S. Department of Energy under Contract DE-AC06-96RL13200

Fluor Hanford

P.O. Box 1000

Richland, Washington 
SNF-6218

Revision 0

EDT 628538

\section{Basler Electric BEI-51/27R U3E-Z3P- B1C1F Overcurrent Relay}

C Van Katwijk

$\mathrm{FH}$

Date Published

April 2000

Prepared for the U.S. Department of Energy

Assistant Secretary for Environmental Management

Project Hanford Management Contractor for the

U.S. Department of Energy under Contract DE-AC06-96RL13200

Fluor Hanford

P.O. Box 1000

Richland, Washington
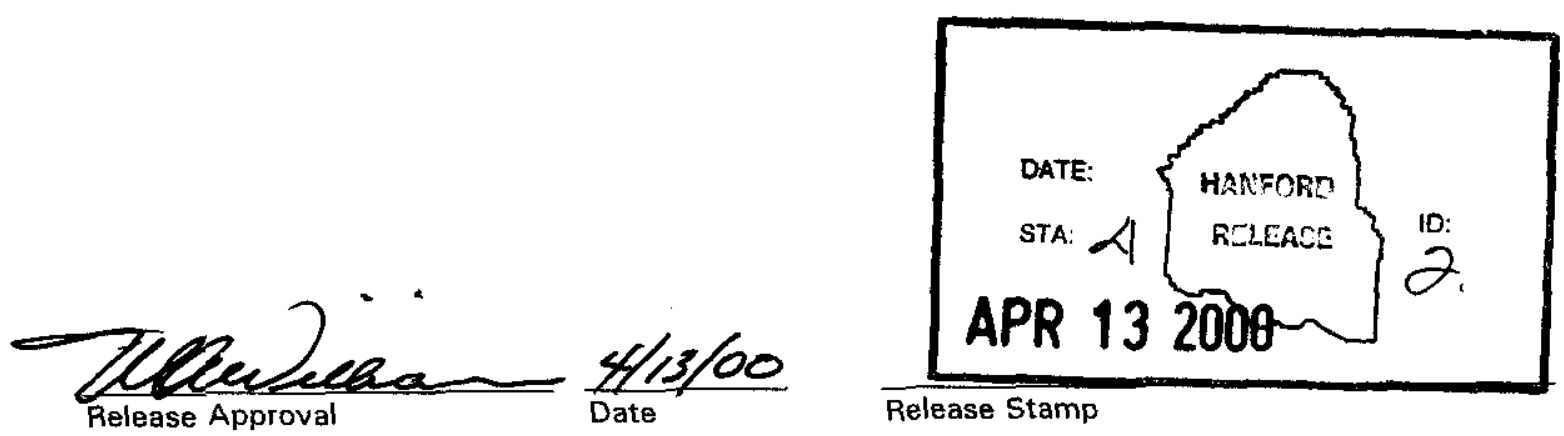
TRADEMARK DISCLAIMER

Reference herein to any specific commercial product, process, or service by trade name, trademark, manufacturer, or otherwise, does not necessarily constitute or imply its endorsement, recommendation, or favoring by the United States Government or any agency thereof or its contractors or subcontractors.

This report has been reproduced from the best available copy.

Printed in the United States of America

Total Pages: $\quad 10$

SNF- 6218, Revo 


Commercial Grade Item Upgrade Dedication Form
\begin{tabular}{|l|l|}
$\begin{array}{l}\text { ECN No. N/A CGI No. CGI-SNF-D-20-1-C1-065 } \\
\text { Titte: Basler Electric BEI-51/27R U3E-Z3P.B1C1F Overcurrent Relay }\end{array}$ & Page 1 of 8 \\
\hline
\end{tabular}

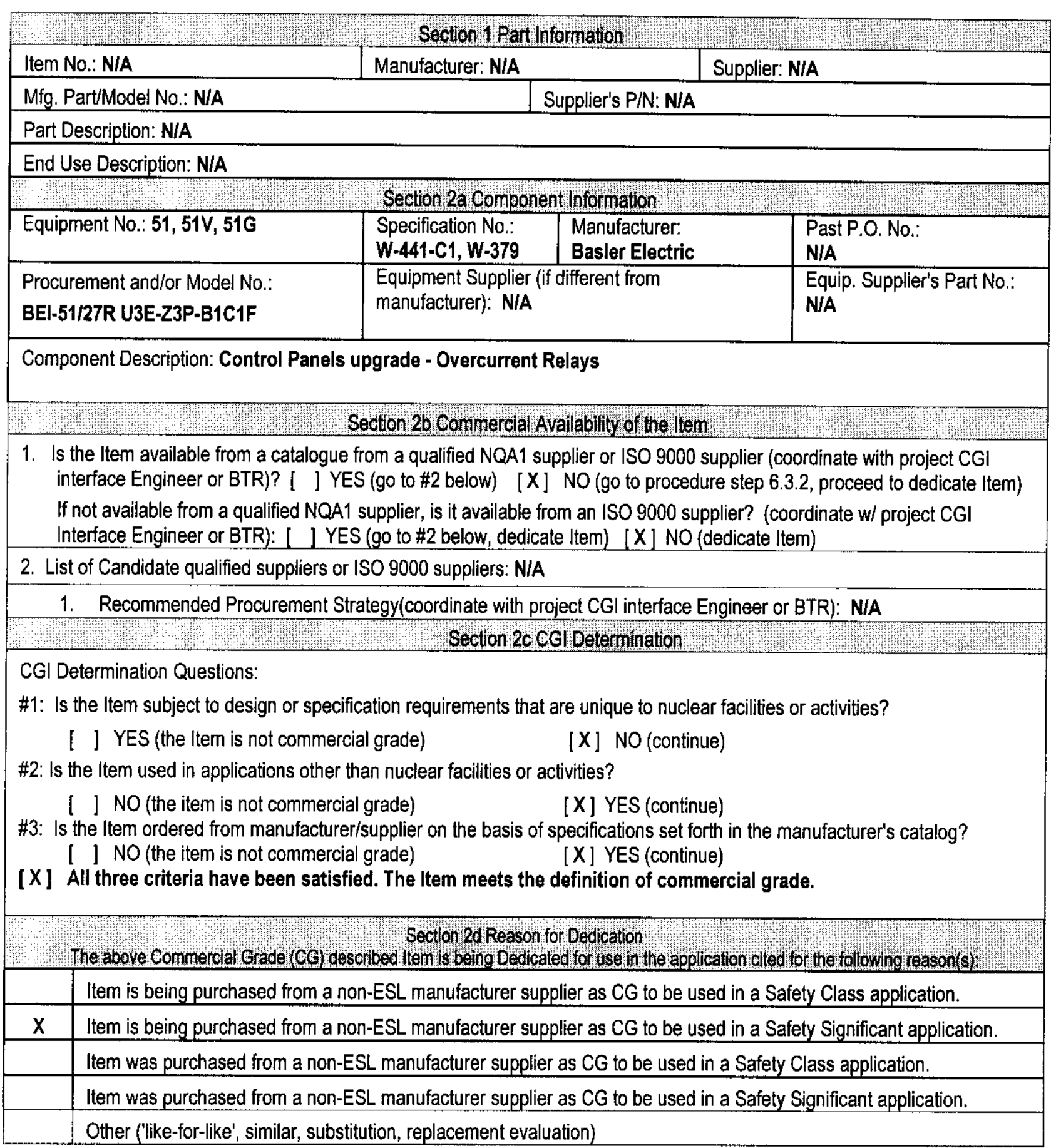


Commercial Grade Item Upgrade Dedication Form

ECN No. N/A CGINo. CGI-SNF-D-20-1-C1-065

SNF-6218, Rev. 0

Title: Basler Electric BEl-51/27R U3E-23P.B1C1F Overcurrent Relay

Page 2 of 8

\section{Section 8 Fallure Effects Evaluation}

A. PartComponent Safety Function:

1. Maintain overcurrent protection of the control panels

B. Part/Component Functional Mode:

Safety Function \#1:

Safety Function \#2:

Safety Function \#3:

\begin{tabular}{|l|l}
\hline & Active \\
Active \\
Active
\end{tabular}

\begin{tabular}{|l|l|}
\hline$X$ & Passive \\
\cline { 1 - 1 } Passive \\
Passive
\end{tabular}
Active - Mechanical or Electrical change of state is required to occur for the component to perform its safety function

Passive - Change of state is not required for the component to perform its safety function

C. Host Component Safety Function (if applicable): N/A

1.

D. Failure Mode(s) and the effects on component or system safety function (see Worksheet 1):

1. External events that causes structural component failure

2. Seismic 3/1 Protection for adjacent SS SSCs -Containment

_u Secion 4 Environmental \& Natural Phenomena Hazard Design

Environmental Qualification Required:

$\begin{array}{ll}\text { Yes } & \\ & \mathrm{x}\end{array}$

Environmental Condition A

Natural Phenomena Hazard (NPH) Design Required:

$\begin{array}{lll}\text { Yes } & X \\ \text { No } & & \\ & & \end{array}$
If yes: Environmental Qualification Requirements

Limiting Environmental Conditions:

Required Safety Functions:

Qualification Period:

If yes: NPH Design Requirements

Peformance Category: PC-2

NPH Design Req'ts.:

Required Safety Functions: Seismic 3/1 Protection for adjacent SS SSCs - Containment

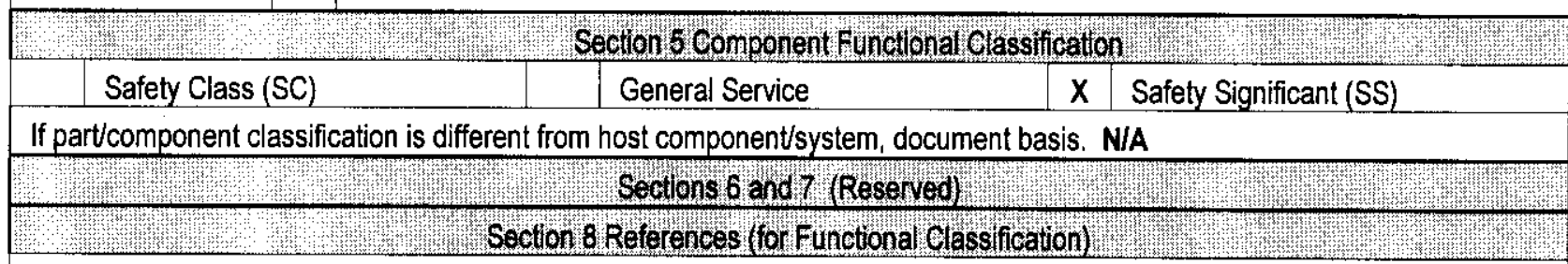

National Codes/Standards: NEC

Safety Analysis Report (SAR): HNF- 3553, Rev. 0, Annex B

Drawings: Nutherm 59729

Vendor Manual/Manufacturer/Supplier Information: Catalog Cut Sheets - Basler Electric 


\begin{tabular}{|c|c|}
\hline Commercial Grade Item Upgrade Dedication Form & SNF-6218, Rev. 0 \\
\hline ECN No. N/A CGINo. CGI-SNF-D-20-1-C1-065 & Page 3 of 8 \\
\hline
\end{tabular}

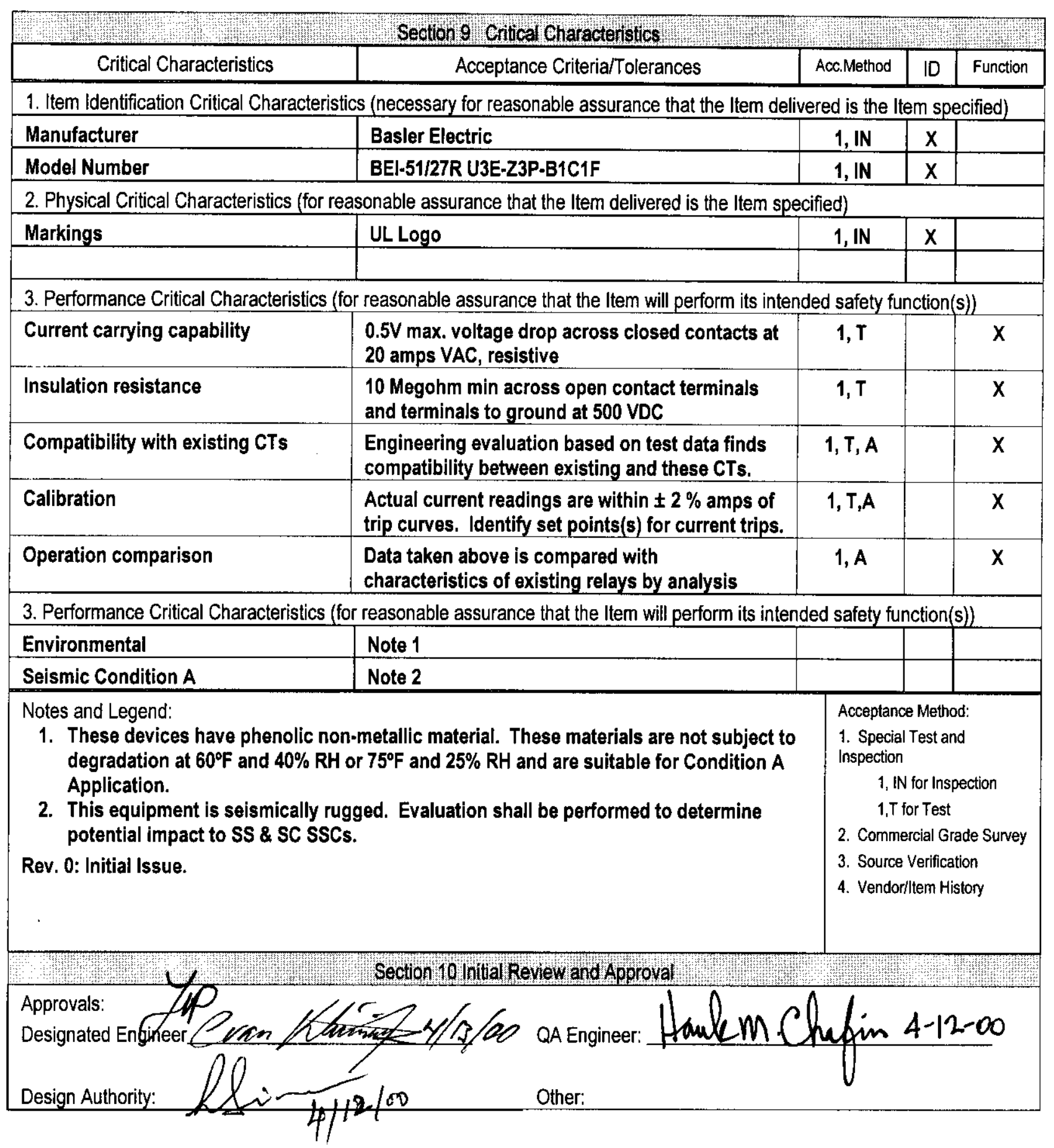




\begin{tabular}{|l|l|l}
\hline ECN No. NIA CGINo. CGI-SNF-D-20-1-C1-065 & Page 4 of 8
\end{tabular}

Title: Basler Electric BEl-51/27R U3E.Z3P-B1C1F Overcurrent Relay

\section{WORKSHEET 1}

\section{DETERMINATION OF FAILURE MECHANISMS}

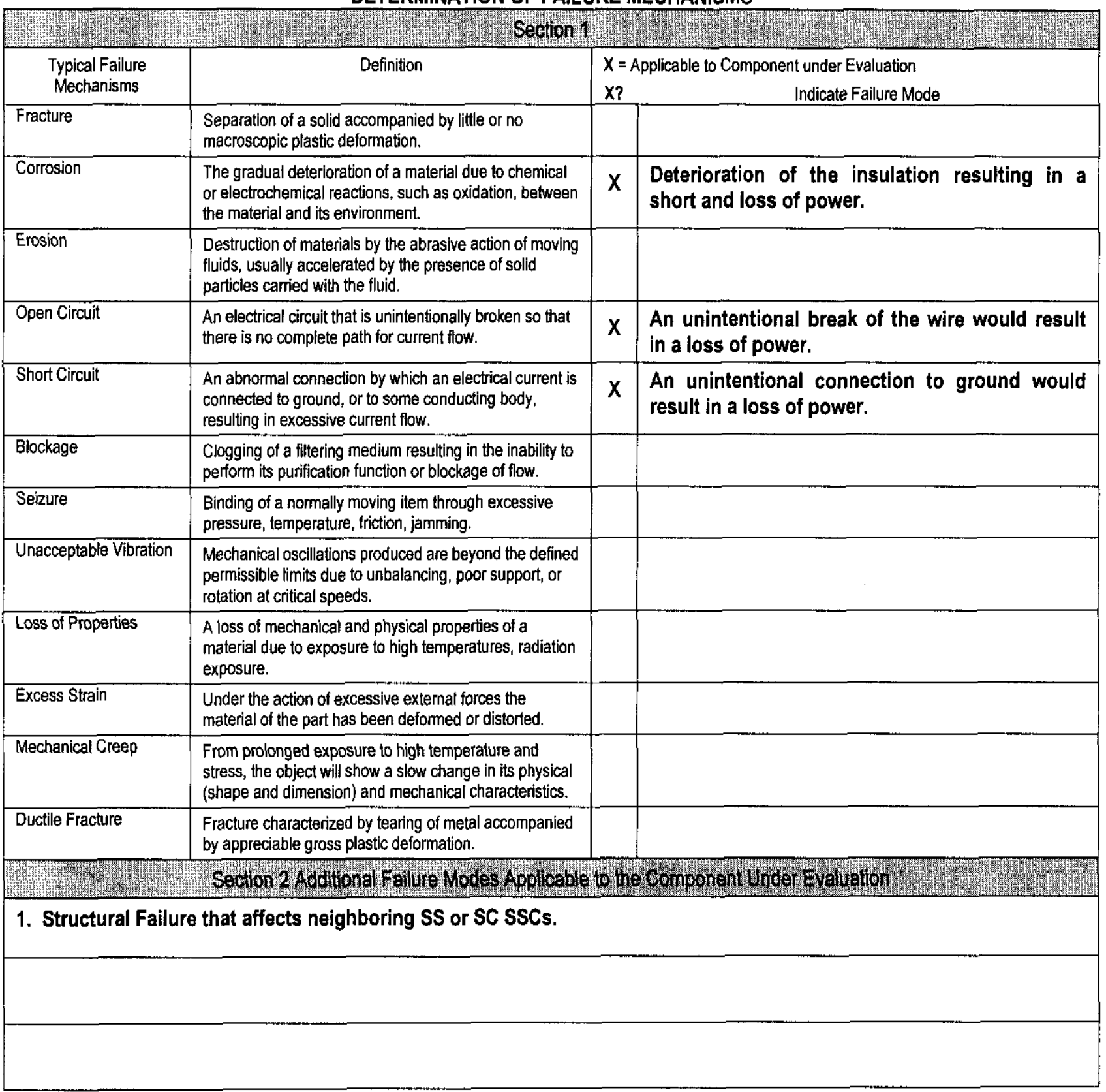


Checklist 1 - Acceptance Method 1 - Special Test/Inspection Verification

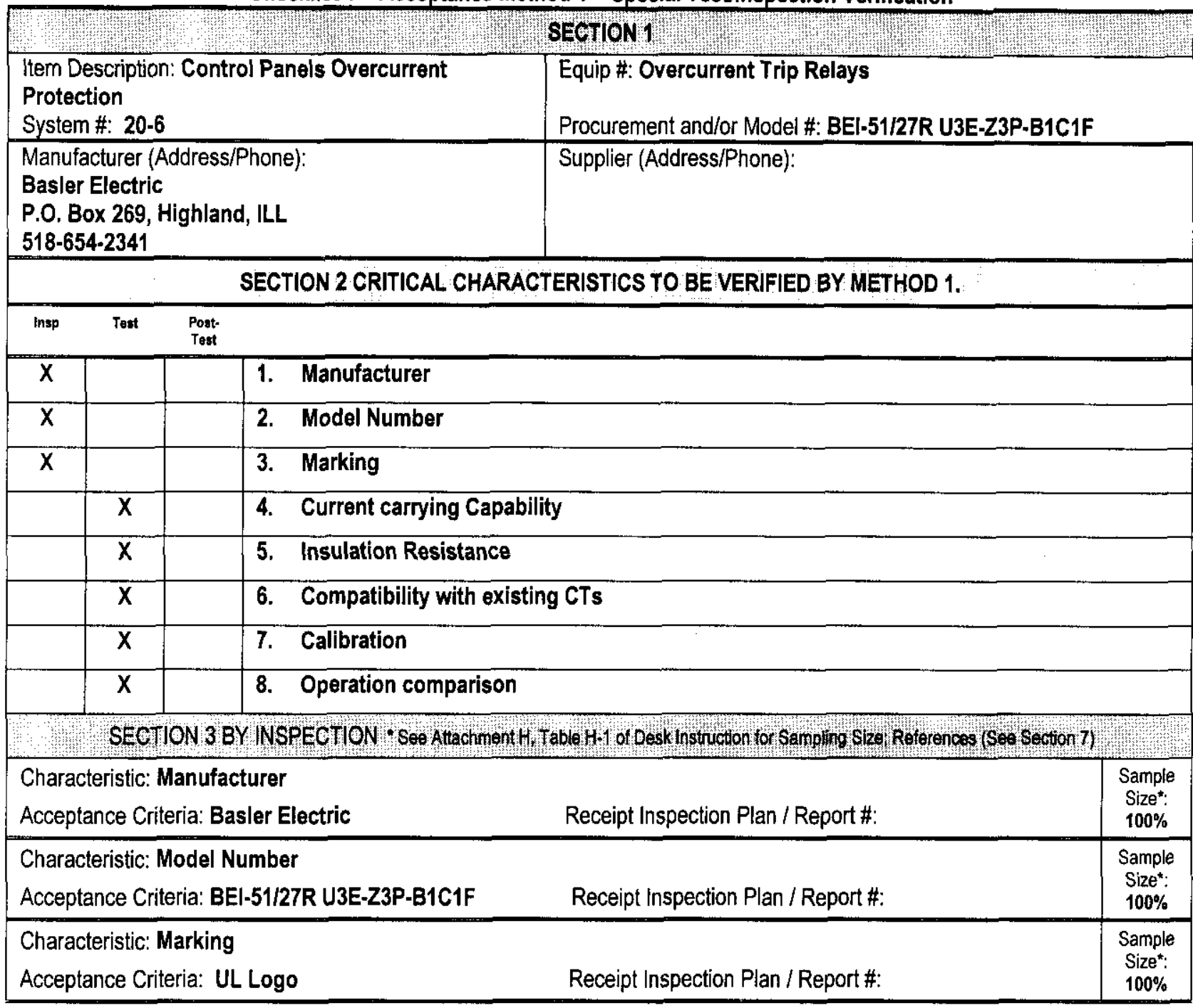




\begin{tabular}{|c|c|}
\hline Commercial Grade Item Upgrade Dedication Form & SNF-6218, Rev. 0 \\
\hline $\begin{array}{l}\text { ECN No. N/A CGI No. CGI-SNF-D-20-1-C1-065 } \\
\text { Title: Basler Electric BEl-51/27R U3E-Z3P-B1C1F Overcurrent Relay }\end{array}$ & Page 6 of 8 \\
\hline
\end{tabular}

\begin{tabular}{|c|c|}
\hline Characteristic for Test: Current carrying Capability & Samp Size*: [X]Normal[ ]Reduced[ ]Tightened \\
\hline \multicolumn{2}{|c|}{ Acceptance Criteria: $0.5 \mathrm{~V}$ max. voltage drop across closed contacts at 20 amps VAC, resistive } \\
\hline Actual Test Value: & Test Plan and Report\#: \\
\hline Characteristic for Test: Insulation Resistance & Samp Size*: [X]Normall ]Reduced[ Jightened \\
\hline \multicolumn{2}{|c|}{ Acceptance Criteria: 10 Megohms min across open contact terminals and terminals to ground (500VDC megger) } \\
\hline Actual Test Value: & Test Plan and Report\#: \\
\hline Characteristic for Test: Compatibility with existing CTs & Samp Size: [X]Normall ]Reduced[ ]Tightened \\
\hline \multicolumn{2}{|c|}{ Acceptance Criteria: Engineering evaluation based on test data finds compatibility between existing and these CTs. } \\
\hline Actual Test Value: & Test Plan and Report \#: \\
\hline Characteristic for Test: Calibration & Samp Size": [X]Normall ]Reduced[ Jightened \\
\hline \multicolumn{2}{|c|}{ Acceptance Criteria: Actual current readings are within $\pm 2 \%$ amps of trip curves. Identify set points(s) for current trips. } \\
\hline Actual Test Value: & Test Plan and Report\#: \\
\hline Characteristic for Test: Operation comparison & Samp Size: [X]Normal[ ]Reduced] JTightened \\
\hline \multicolumn{2}{|c|}{ Acceptance Criteria: Data taken above is compared with characteristics of existing relays by analysis } \\
\hline Actual Test Value: & Test Plan and Report \#: \\
\hline
\end{tabular}

**If Supplier/Manufacturer or Other, Refer to CGI Checklist-2 for Support Information 


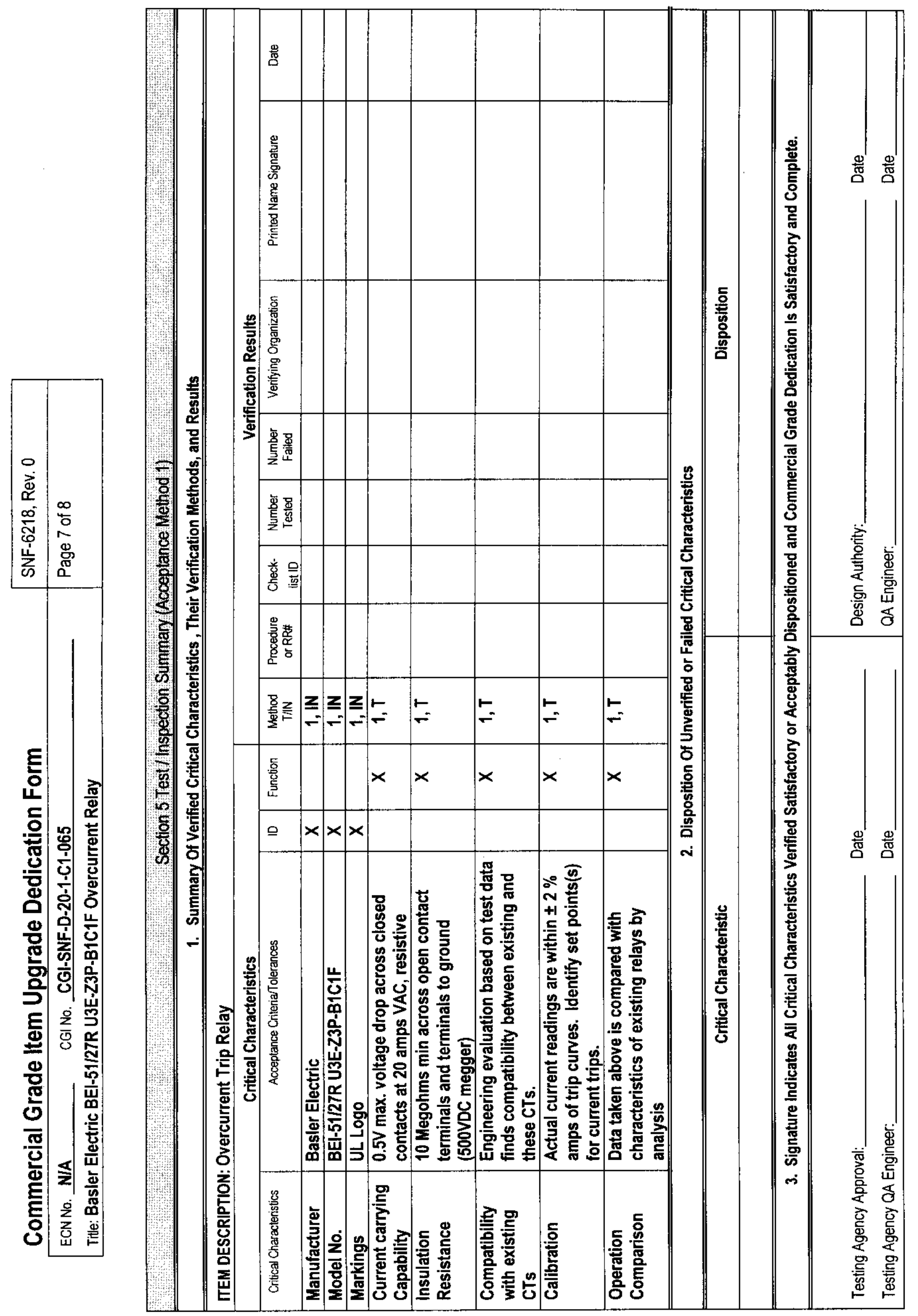


Commercial Grade Item Upgrade Dedication Form

ECN No. N/A CGINo. CGI-SNF-D-20-1-C1-065

SNF-6218, Rev. 0

Titte: Basler Electric BEI-51/27R U3E-Z3P-B1C1F Overcurrent Relay

Page 8 of 8

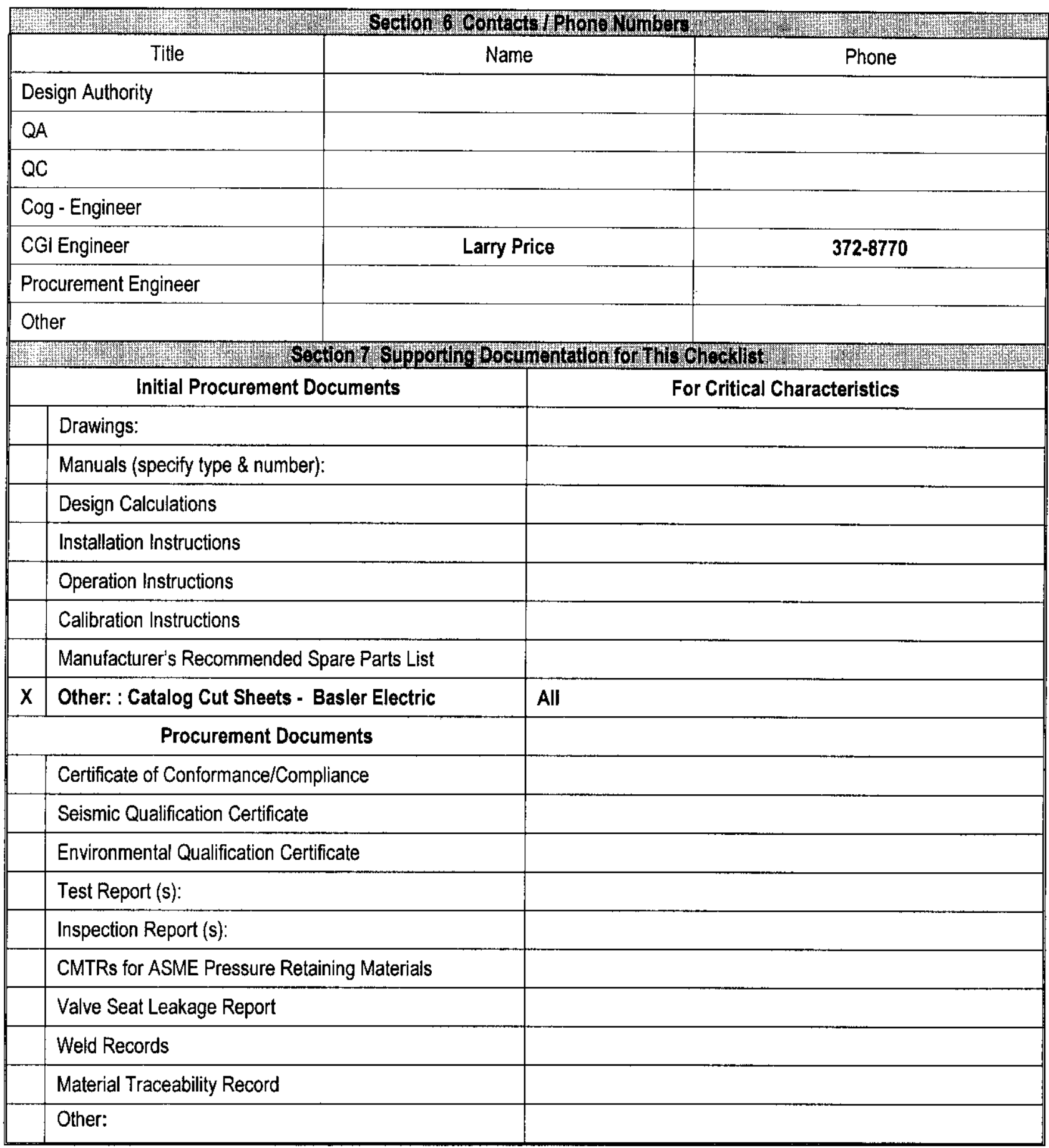




\section{DISTRIBUTION SHEET}

To

Distribution

Project Title/Work Order

W-441, SNF-6217 and SNE-6218, Rev. 0
From

T. Nuxall, SNE-CVD

Page 1 of 1

Date $4 / 13 / 00$

EDT No. 628538

ECN No. N/A

Name

D. Whitehurst

G. Singh

A. Artzer (CVD Library)

R. Ramsgate

J. Brehm

P. Beaudet

P. Morrell (AVS)

M. Evarts (AI)

L. Price

SNE Startup

SNF Project Files

SNE Satelite Library

C. Van Katwijk

D. Whitworth

T. Nuxall

C. Miska

\begin{tabular}{|c|c|c|c|c|}
\hline MSIN & $\begin{array}{c}\text { Text } \\
\text { With All } \\
\text { Attach. }\end{array}$ & Text Only & $\begin{array}{l}\text { Attach./ } \\
\text { Appendix } \\
\text { Only }\end{array}$ & $\begin{array}{c}\text { EDT/ECN } \\
\text { Only }\end{array}$ \\
\hline
\end{tabular}

